\title{
Analysis of lung volume restriction in patients with respiratory muscle weakness
}

\author{
ANDRÉ DE TROYER, SAMUEL BORENSTEIN, AND RENÉ CORDIER \\ From the Chest Service, Erasme University Hospital, and the Brain Research Unit, Brussels School of \\ Medicine, Brussels, Belgium
}

ABSTRACT We investigated pulmonary mechanics in 25 patients, 9 to 55 years of age, with a variety of generalised neuromuscular diseases and variable degrees of respiratory muscle weakness. The average degree of inspiratory muscle force was $39.2 \%$ (range $8-83 \%$ ) of predicted. The lung volume restriction far exceeded that expected for the degree of muscle weakness: the observed decrement in respiratory muscle force should, theoretically, decrease vital capacity to $78 \%$ of its control value, while the mean VC in our patients was only $50 \%$ of predicted. Analysis of lung pressure-volume curves indicated that the two principal causes of the disproportionate loss of lung volume were a reduction in lung distensibility probably caused by widespread microatelectasis, and a decrease in the outward pull of the chest wall. Because it reflects both direct (loss of distending pressure) and secondary (alterations in the elastic properties of the lungs and chest wall) effects of respiratory muscle weakness on lung function, we conclude that, in these patients, the vital capacity remains the most useful measurement to follow evolution of the disease process or response to treatment.

Respiratory muscle weakness is a frequent occurrence in many neuromuscular diseases. Although it is well established that weakness of the respiratory muscles produces a restrictive ventilatory defect, the precise relationship between the degree of, muscle weakness and the type of lung dysfunction is still unclear. In particular, there is disagreement as to whether the resting end-expiratory position decreases, ${ }^{1-4}$ increases, ${ }^{5}$ or remains unchanged. ${ }^{6-8}$ Similarly, some investigators have reported no change in residual volume, ${ }^{239}$ while a majority of studies have found an increase in this volume. ${ }^{5-8}$ An additional problem concerns the loss of inspiratory capacity associated with the impairment of inspiratory muscle function. Indeed, because of the shape of the maximal static pressure-volume curves of the respiratory system, a large degree of respiratory muscle weakness would be predicted to have only a moderate effect on lung volume. However, recent data suggest that, in patients with respiratory muscle weakness, the relationship between loss of respiratory muscle force and loss of lung volume is not always predictable, in that the decrease

Address for reprint requests: Dr A De Troyer, Chest Service, Erasme University Hospital, 808 Route de Lennick, 1070 Brussels, Belgium. in lung volume is often disproportionate to the loss of muscle force. ${ }^{210}$ Similar findings have been made in normal volunteers in whom acute weakness of the respiratory muscles produced by partial curarisation was accompanied by large changes in lung volume, changes that far exceeded the predictions of the effects of muscle weakness on lung volume. ${ }^{11}$

We recently investigated pulmonary mechanics in 25 patients with a variety of generalised neuromusoular disonders and a wide range of respiratory muscle performance. The three main purposes of the present report are: (1) to delineate the pattern of pulmonary function associated with respiratory musole weakness; (2) to evaluate the lung volume restriction associated with a chronic involvement of the respiratory muscles, with particular emphasis on the relationship between loss of respiratory muscle strength and loss of lung volume; and (3) to determine the factors, other than the weakness itself, that are mainly involved in the lung volume restriction.

\section{Patients}

Twenty-five consecutive patients with generalised neuromuscular disorders and referred to our 
laboratory for evaluation of respiratory muscle force form the basis of the report. The group consisted of 19 males and six females who had a mean age of 26 years, with a range of 9 to 55 years. Eighteen patients had miscellaneous myopathies, among which 14 had Duchenne-type muscular dystrophy, two had limb-girdle dystrophy, and two had muscular dystrophy of the facioscapulohumeral type. Four patients had muscular wasting associated with dystrophia myotonica. The remaining three patients had respiratory muscle weakness caused by polymyositis, previous poliomyelitis, and GuillainBarré syndrome, respectively. The diagnoses were based on clinical and appropriate laboratory examination including serum enzymes, nerve conduction studies, electromyograms, and, in some cases, muscle biopsies. All patients but two were lifelong nonsmokers, but none had a history of chronic obstructive lung disease or clinical evidence of congestive heart failure. Only two patients complained of dyspnoea at rest at the time of the study. Four of the 25 patients, all of whom had progressive muscular dystrophy, had radiographic shadowing suggesting partial atelectasis of one lobe on the day of the pulmonary evaluation.

\section{Methods}

All measurements were carried out with the subject seated in a constant volume body plethysmograph. Lung volumes, maximal expiratory and inspiratory flow-volume curves, and specific airway conductance were obtained by standard methods described previously. ${ }^{12}$

Expiratory pressure-volume (PV) curves were obtained by a quasi-static method ${ }^{13}$ with an oesophageal latex balloon (length $10 \mathrm{~cm}$; perimeter $3.5 \mathrm{~cm}$; air volume $0.4 \mathrm{ml}$ ) introduced via the nose into the oesophagus. A marker was placed on the polyethylene tubing exactly $42 \mathrm{~cm}$ from the balloon tip and balloon adjustment began when this marker appeared at the external nares. Recording of the curves was preceded by three full inflations to assure a constant volume history. Lung volume was plotted versus transpulmonary pressure on a direct-writing $X-Y$ recorder. Several deflational PV curves were obtained in each patient and a line of best fit was drawn by eye through at least three sets of $\mathrm{PV}$ data that agreed to $\pm 1 \mathrm{~cm} \mathrm{H}_{2} \mathrm{O}$. The static recoil pressure of the lung (Pst (1)) was measured at various percentages of total lung capacity (TLC) that was calculated by adding to FRC the mean inspiratory capacity measured during the $\mathrm{X}-\mathrm{Y}$ recording of the PV curves. $\overrightarrow{\vec{F}}$ Static expiratory lung compliance was measuredo as the slope of the PV curve over the 0.5 litreo above functional residual capacity (FRC). The same technique was used for obtaining ex- 0 piratory $P V$ curves in the children of the presento study, except for the distance between ballon tip? and nares. ${ }^{13}$

Minimal (inspiratory) pleural pressures ( $\mathrm{P} p \dot{L}$ min) were obtained by repeated measurements afs various lung volumes while the subject attempted maximal inspiratory efforts against an obstruc ${ }_{\omega}^{x}$ tion. A conventional mouthpiece and noseclip were used, and pressures sustained for one secono were recorded. At least 15 maximal efforts were् recorded on each patient, and the lowest values at each lung volume were used to construct curves of minimal pleural pressure against lungs volume.

Predicted values for lung volumes are fromw Amrein et al. ${ }^{14}$ Predicted values for the $P V \vec{L}$ curves and $\mathbf{P}$ pl min are derived from resultso obtained in 40 healthy children ${ }^{13}$ and 135 normal subjects studied in our laboratory. ${ }^{15}$

\section{Results}

In 23 of the 25 patients, the shape of the $P$ p min-volume curve was similar to that seen if normal subjects - it was curvilinear and the lowest pressures were achieved at or near FRC? Minimal pleural pressure at FRC ranged fror -19 to $-86 \mathrm{~cm} \mathrm{H}_{2} \mathrm{O}$. However minimal pleurat pressures are highly dependent on the lung volume at which they are measured, as de्es veloped in a previous study. ${ }^{15}$ Therefore, the lung volume restriction present in our patients. had to be taken into account before comparisod was made with the normal predicted values. After the correction has been made for the los of lung volume, the degree of inspiratory musch force ranged between 8 and 83 (mean $\pm \mathrm{SE}$. $39.2 \pm 3.6) \%$ of predicted. The mean ourve obtained in our patients is compared to the mean predicted one in fig 1 .

Average values of lung volumes are given table 1. The degree of lung volume restriction varied from 30 to $101 \%$ of predicted TLC and 14 to $93 \%$ of predicted VC. The average decreas in VC was $50 \%$ and TLC $33 \%$. The FRC w\& below the normal range in most of the patientst the mean predicted value averaged 2.75 litres compared to $2 \cdot 16$ litres in the patients, a sign? ficant decrease $(\mathrm{p}<0.001)$. A significant positi relationship was found between FRC and the inspiratory muscle force (fig 2 , left panel). The 


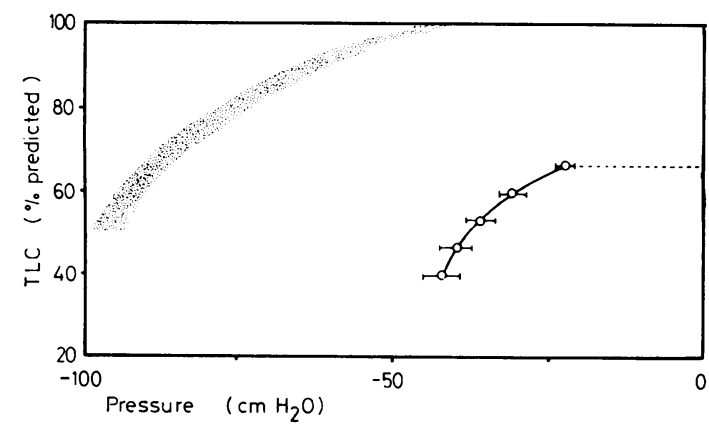

Fig 1 Volume-minimal pleural pressure curves obtained in 25 patients with respiratory muscle weakness; mean $\pm S E$ values are shown. Shaded area represents predicted values. Volume expressed as a percentage of predicted $T L C$.

Table 1 Subdivisions of lung volume (mean $\pm S E$ ) in 25 patients with neuromuscular disorders

\begin{tabular}{lclll}
\hline Subjects & $V C(l)$ & $F R C S(l)$ & $R V(l)$ & $T C L(l)$ \\
\hline Patients & & & & \\
Mean & 1.89 & 2.16 & 1.48 & 3.41 \\
SE & 0.16 & 0.15 & 0.11 & 0.24 \\
Predicted & & & & \\
Mean & 3.80 & 2.75 & 1.39 & 5.10 \\
SE & 0.22 & 0.18 & 0.10 & 0.31 \\
$t$ value & 8.67 & 5.03 & 1.15 & 6.87 \\
p value & $<0.001$ & $<0.001$ & NS & $<0.001$ \\
\hline
\end{tabular}

The predicted values for lung volumes are those of Amrein et al. ${ }^{14}$

RV was not significantly altered.

The mean deflational PV curve of the lung obtained in the 25 patients is compared to the predicted one in fig 3. The graph demonstrates clearly that the PV curve of the patients was reduced on its volume axis. For any given transpulmonary pressure there was a marked decrease in absolute lung volume. Both lung recoil pressure at full inflation and static ex-

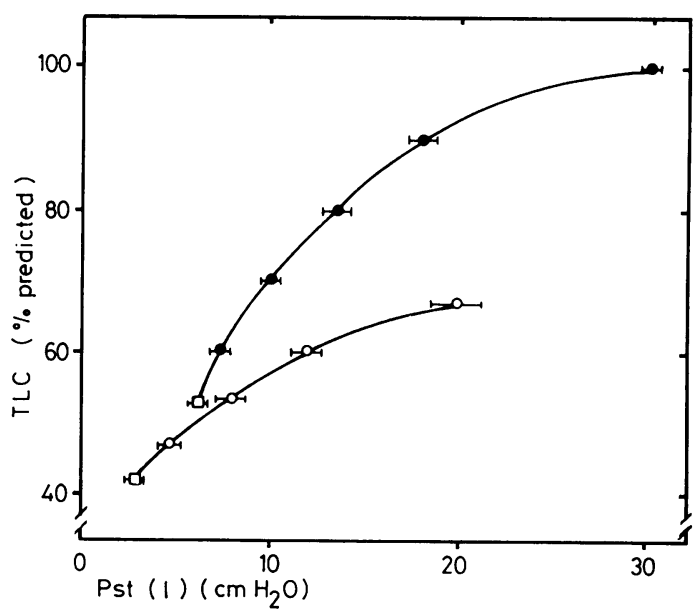

Fig 3 Static expiratory pressure-volume curve of the lung in patients with neuromuscular disorders and respiratory muscle weakness; average data of 25 patients (open circles). Volume expressed as percentage of predicted TLC and closed circles represent mean predicted values. Each bar represents \pm 1 SEM. Pst $(l)=$ static lung recoil pressure. The observed and predicted resting lung volumes are also indicated (open squares).

piratory compliance were significantly reduced (table 2). The average decrease in lung compliance was $40 \%$. However, when volume was expressed as a fraction of measured, rather than predicted, TLC, the mean curve obtained in the patients was parallel to the predicted one, especially in its linear portion (fig 4). The transpulmonary pressure at FRC was below normal in 23 of the 25 patients and it was very low in some cases. The mean observed value averaged $2.8 \mathrm{~cm} \mathrm{H}_{2} \mathrm{O}$, compared to a mean predicted value of $6.6 \mathrm{~cm} \mathrm{H}_{2} \mathrm{O}$, a significant decrease

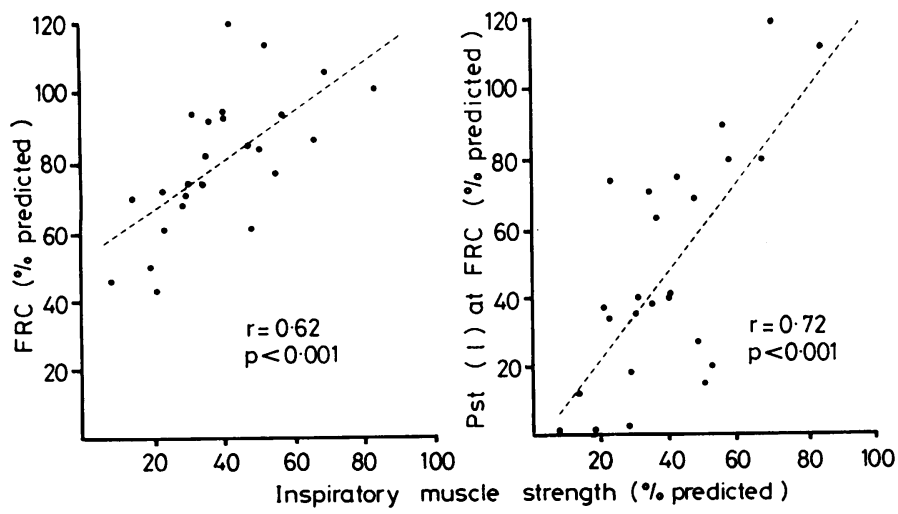

Fig 2 Relationships between inspiratory muscle force and FRC (left panel) and between inspiratory muscle force and static recoil pressure of the lung (Pst (l)) at FRC (right panel) in 25 patients with respiratory muscle weakness. Dashed lines are the lines of regression. 
Table 2 Data from lung pressume-volume studies in 25 patients with neuromuscular diseases

\begin{tabular}{|c|c|c|c|}
\hline Subjects & $\begin{array}{l}\text { Static expiratory } \\
\text { compliance } \\
\left(\mathrm{I} / \mathrm{cm} \mathrm{H}_{2} \mathrm{O}\right)\end{array}$ & $\begin{array}{l}\text { Pst (l) at TLC } \\
\left(\mathrm{cm} \mathrm{H}_{2} \mathrm{O}\right)\end{array}$ & $\begin{array}{l}\text { Pst (l)at FRC } \\
\left(\mathrm{cm} \mathrm{H}_{2} \mathrm{O}\right)\end{array}$ \\
\hline \multicolumn{4}{|l|}{ Patients } \\
\hline Mean & $0 \cdot 113$ & $19 \cdot 9$ & $2 \cdot 8$ \\
\hline SE & 0.017 & $1 \cdot 2$ & 0.4 \\
\hline \multicolumn{4}{|l|}{ Predicted } \\
\hline Mean & $0 \cdot 188$ & $30 \cdot 1$ & $6 \cdot 6$ \\
\hline SE & 0.017 & 0.6 & 0.4 \\
\hline$t$ value & $6 \cdot 11$ & $6 \cdot 27$ & $6 \cdot 48$ \\
\hline p value & $<0.001$ & $<0.001$ & $<0.001$ \\
\hline
\end{tabular}

The predicted values are derived from measurements performed in 40 healthy children 13 and 120 healthy adults 15 studied in this laboratory

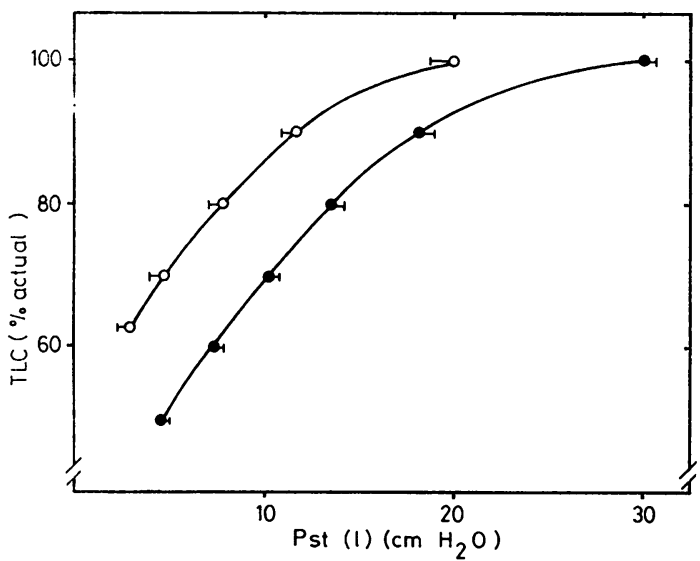

Fig 4 Same data as in fig 3, with lung volume expressed as a percentage of actual rather than predicted $T L C$.

$(p<0.001)$ (table 2). The Pst (1) at FRC correlated positively and significantly with the loss of inspiratory muscle force $(r=0.72 ; p<0.001)$ : the weaker the inspiratory muscles, the lower the lung recoil pressure at resting lung volume (fig 2, right panel). Finally, in none of the patients, was there any evidence of airway obstruction. Specific airway conductance was within normal limits or above the normal upper limit of $0.371 / \mathrm{s} / \mathrm{cm} \mathrm{H}_{2} \mathrm{O}$ in all cases, and the maximum expiratory flow-static recoil relationship was within the normal range in each patient.

The four patients who had radiologically visible atelectasis were among those who had the smallest lung volumes and the lowest values of lung compliance. However, a similar degree of pulmonary dysfunction was present in some patients who had clear radiographs, and the functional disturbances were qualitatively identical in all the patients in this study, regardless of chest radiograph or type of underlying disease.

\section{Discussion}

Weakness of the inspiratory muscles was present at a variable degree, in most of the 25 patients studied here. In 19 of them the minimal pleura pressures were less than $50 \%$ of predicted, which indicates that severe respiratory muscle weakness is common in patients with generalised neuro? muscular diseases.

Our findings confirm that patients wits respiratory muscle weakness have a restrictive ventilatory impairment. In addition, they show that in these patients, the lung volumes are reduced in approximate proportion to the reduction of the pressures developed durin 8 maximum static inspiratory efforts. The finding of large changes of lung volume was unexpected: because of the shape of the pressure-volums characteristic of the relaxed respiratory systen which becomes curvilinear only near the exco tremes of the vital capacity, lung volume should be well preserved even in the face of severeo muscle weakness. For example, if it is assumed that the relaxation characteristic of the chest wall in the patients was normal, it can bs calculated from our standard maximal statio pressure-volume diagram ${ }^{15}$ that a decrement of inspiratory muscle strength identical to that observed here at FRC would, theoretically $\overrightarrow{6}$ decrease TLC to only $86.5 \%$ of its control value (fig 5). The observed data strongly differ from this prediction, since TLC in our patients was. on an average, $67 \%$ of predicted. The fact that

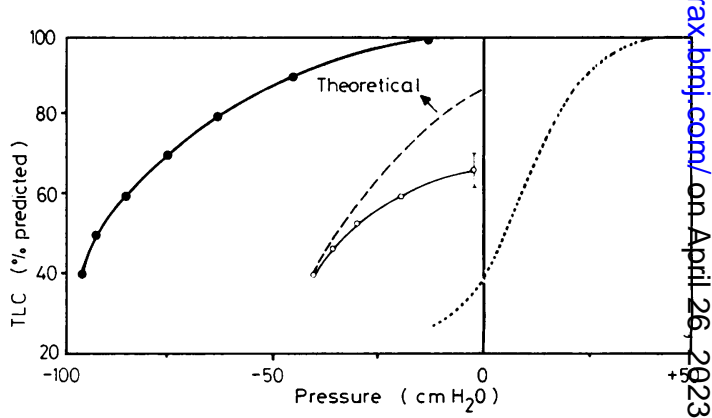

Fig 5 Comparison between observed (open circles) and theoretically predicted (dashed line) maximal static inspiratory pressure-volume relationship of the respiratory system in patients with chronic respiratory muscle weakness. The normal curve (closed circles) is drawn from the data obtained in 135 healthy subjects. ${ }^{15}$ The predicted relationship was constructed assuming a decrement in muscle pressure at all lung volumes proportional to that measured at FRC. Dotted line is the normal relaxation $P V$ relationship of the respiratory system 
the loss of lung volume was out of proportion to that anticipated for the degree of muscle weakness is also illustrated in fig 6 . The solid curve in this graph is the theoretical relation between VC and respiratory muscle strength, calculated from the standard maximal static pressure-volume diagram, and assuming again that respiratory system recoil for patients with chronic weakness of the respiratory muscles is the same as that measured in normal subjects, and that there is a uniform involvement of the inspiratory and expiratory muscles. Based on these theoretical considerations, a $61 \%$ decrease in respiratory muscle strength would be associated with a VC of $78 \%$. The graph shows also the individual values obtained in our patients and the logarithmic regression calculated from these values. As expected, the VC was highly and positively correlated with the inspiratory muscle strength $(\mathrm{r}=0.88 ; \mathrm{p}<0.001)$, but the relationship was shifted downwards when compared to the theoretical one-that is, the VC in the patients was clearly lower, at any level of respiratory muscle force, than would be predicted on the basis of normal respiratory system recoil. These considerations show that the loss of lung volume in patients with a chronic involve-

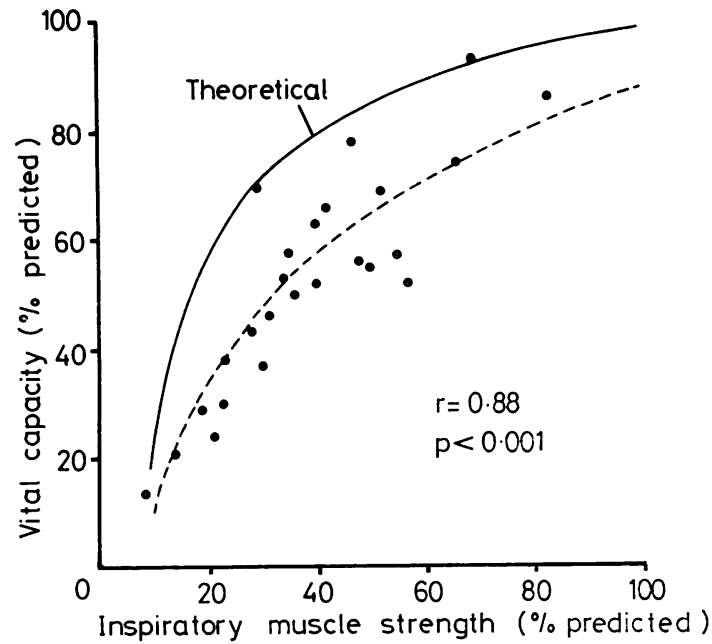

Fig 6 The solid curve indicates the theoretical effect of respiratory muscle weakness on vital capacity constructed by assuming (1) that the relaxation $P V$ characteristic of the chest wall is normal, and (2) that the inspiratory and expiratory muscles are involved uniformly. The dashed curve is the logarithmic regression calculated from the data obtained in the 25 patients with various neuromuscular diseases (closed circles). ment of the respiratory muscles is disproportionate to the loss of respiratory muscle force and thus suggest that factors other than weakness itself are implicated in the lung volume restriction. We believe these discrepancies between theoretical considerations and experimental findings are explained by changes in lung elasticity and by alterations in the passive recoil of the ohest wall.

Static expiratory lung compliance was markedly decreased in our patients. Most clearly, this reduction far exceeded the effects that could result from the only difference in lung volume history, and values of compliance obtained during inflation were also reduced. This implies that patients with a longstanding involvement of the respiratory muscles have alterations in the elastic properties of the lung. Indeed, if the loss of distending pressure were the only effect of muscle weakness, the PV curve of the lung would be truncated but compliance measured over the tidal range should be close to normal. Consequently there exists in these patients a parenchymal involvement that must contribute to the loss of lung volume. That the change in lung distensibility is an important determinant of the degree of lung volume restriction is suggested by the finding that there was a clear relationship between TLC and the compliance at FRC (fig 7), as well as between VC and compliance $(r=0.79 ; p<0.001)$. A close relationship between compliance and TLC has already been reported in such patients. ${ }^{16}$

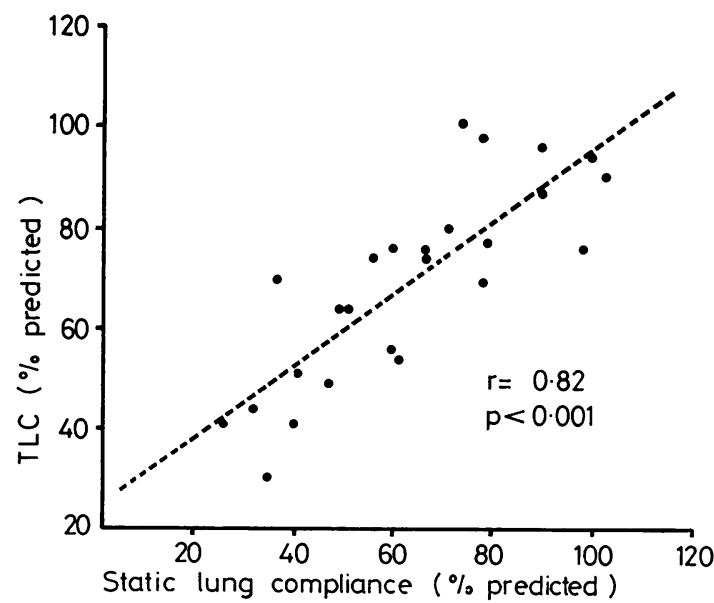

Fig 7 Relationship betwen static lung compliance $\left(C_{L}\right)$ and total lung capacity $(T L C)$ in 25 patients with respiratory muscle weakness. Dashed line is the line of regression. 
The present observations of markedly decreased lung compliance and increased lung recoil pressure in patients with respiratory muscle weakness are consistent with those of Gibson et al. ${ }^{2}$ These workers pointed out that these changes were similar to those seen in normal subjects in whom expansion is prevented by strapping of the chest wall. ${ }^{17} 18$ Because of this similarity and although they felt atelectasis was the most likely explanation, they discussed the possibility that some alterations in the surface properties of the alveolar lining layer, related to the low volume breathing, was responsible for these changes. This possibility is not supported by the observation that the mean PV curve in our patients was quite parallel to the predicted normal when lung volume was related to the measured TLC. The value of relating volume to the measured rather than predicted TLC has been recently discussed by Gibson and Pride $^{19}$ in their study of patients with severe fibrosing alveolitis. They reasoned that in these lungs, the loss of gas-containing alveoli was responsible for most of the changes in the PV curve, and suggested that expressing volume as a percentage of measured TLC would reflect the elastic properties of the surviving ventilated alveoli. This view has been supported by recent measurements obtained in dog lungs with diffuse granulomatous disease. ${ }^{20}$ Therefore the present observation that the slope of the PV curve $(\Delta \mathrm{V} / \Delta \mathrm{P})$ was normal when lung volume was related to the measured TLC would suggest that, in patients with longstanding respiratory muscle weakness, the largest number of functioning alveoli retain normal elastic properties. Results of recent studies in this laboratory on normal volunteers during submaximal neuromuscular blockade are also compatible with the concept that weakness of the respiratory muscles, despite the associated low volume breathing, is not associated with alterations in surface forces and does not directly influence the elastic properties of the lung. ${ }^{12}$

The theoretical considerations discussed above between the degree of muscle weakness and the degree of lung volume restriction were based on the assumption that the PV relationship of the relaxed chest wall was normal in patients with respiratory muscle dysfunction. However, analysis of lung pressure-volume data obtained in the region of resting ventilation suggests that the elastic behaviour of the chest wall is altered in these patients. Indeed the FRC was decreased in most cases. Inasmuch as FRC is determined by the balance between lung and chest wall forces, the observed fall in FRC could have resulted froris the increase in the inward pull of the lung, from a decrease in the tendency of the chest wa to recoil outward, or from a combination of ths two. The first possibility is unlikely. Figure $\frac{8}{8}$ illustrates the theoretical relationship betweef FRC and transpulmonary pressure at FRC when the PV characteristic of the chest wall remains unchanged (solid curve): when an increase it lung recoil pressure is the only cause of decreased FRC, the Pst (1) at FRC must increasę By contrast, in most of our patients, this decrease in FRC was associated with a decreaseo not an increase, in Pst (1) at FRC (fig 8). This is strong evidence that the fall in FRC in these patients is caused primarily by a decrease in the (outward) pull of the chest wall. This observation is consistent with other studies that demonstrated that the ability of the chest wall to recoil oure wards decreased in normal volunteers when acute weakness of the respiratory muscles was prob duced by partial curarisation. ${ }^{12}$ Moreover, in oufe patients, a close relationship existed between the inspiratory muscle force and the decrease of bot FRC and Pst (1) at FRC (fig 2), further suggestin that the reduction of the chest wall force

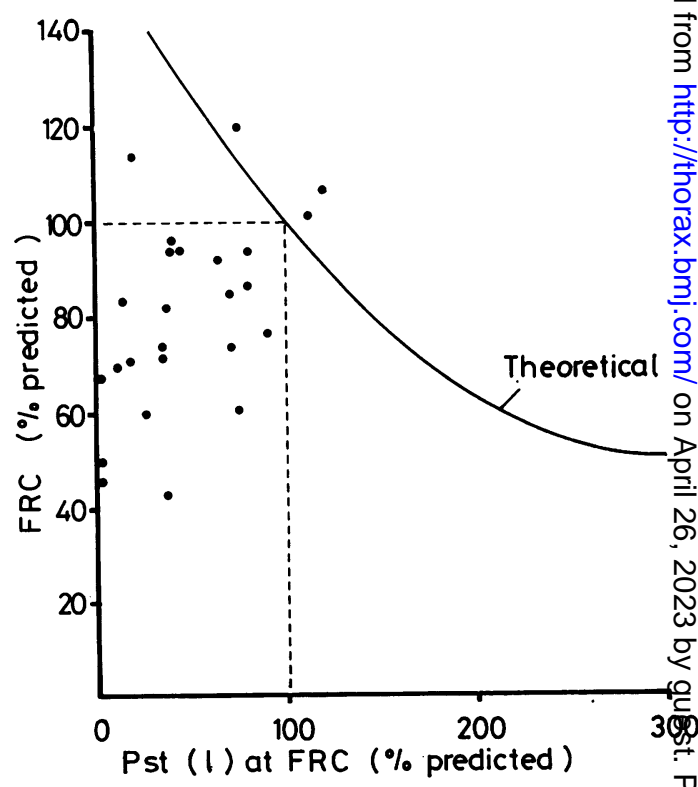

Fig 8 The solid curve indicates the theoretical relationship between $F R C$ and lung static recoil pressure (Pst (l)) at FRC when the elastic recoil of $\frac{\rho}{\mathbb{D}}$ the relaxed chest wall remains normal. The individugit values obtained in 25 patients with respiratory muscle weakness are also shown. 
related to the degree of involvement of the inspiratory muscles. Such a decrease in the outward pull of the chest wall would contribute to the disproportionate loss of lung volume observed in our patients.

The finding of a normal $\mathrm{RV}$ in our patients despite a severe muscle weakness may be explained by at least two factors. Firstly, some patients had radiologically visible atelectasis and, as discussed above, it is likely that atelectasis of a more focal nature, undetectable by chest radiographs, was present in the other cases. Secondly, preservation of $R V$ in the face of decreased expiratory muscle strength may be explained if one considers the determinants of RV. It is well established that the recoil force of the chest wall has a major determining effect on $\mathrm{RV}$ in younger healthy people. ${ }^{21}$ Accordingly, the lack of increase in RV in our patients might be caused by the fact that the decreased expiratory muscle force is counterbalanced by a lesser resistance of the chest wall during expiration.

From these data it would appear that characteristic alterations in lung volumes and respiratory mechanics develop in patients with respiratory muscle weakness. These alterations include reduction in vital capacity, decreased functional residual capacity, normal residual volume, and decreased lung compliance with low lung recoil at resting lung volume. Moreover, it is quite clear from these studies that the change in vital capacity in these patients reflects both direct (loss of distending pressure) and secondary (changes in the passive characteristics of the lungs and chest wall) effects of respiratory muscle weakness on pulmonary function. Measurements of maximal static pressures, in contrast, explore only a limited part of these effects. We conclude, therefore, in disagreement with Braun and Rochester, ${ }^{10}$ that vital capacity, in addition to being the easiest measurement, is the most useful one to perform in these patients to follow evolution of the disease process or response to treatment.

We are indebted to Dr Renoirte for his cooperation in this study, to Miss $M$ Lebrun for technical assistance, and to Miss Douillet for the preparation of the manuscript.

\section{References}

1 Faerber I, Liebert PB, Suskind M. Loss of functional residual capacity in poliomyelitis. $J A p p l$ Physiol 1962; 17:289-92.

2 Gibson GJ, Pride NB, Davis JN, Loh LC. Pulmonary mechanics in patients with respiratory muscle weakness. Am Rev Respir Dis 1977; 115: 389-95.

3 Hapke EJ, Meek JC, Jacobs J. Pulmonary function in progressive muscular dystrophy. Chest 1972; 61:41-7.

4 Vallbona C, Spencer WA. The total lung capacity and its subdivisions in respiratory poliomyelitis. J Chronic Dis 1959; 9:617-35.

5 Inkley SR, Oldenburg FC, Vignos PJ. Pulmonary function in Duchenne muscular dystrophy related to stage of disease. Am J Med 1974; 56:297306.

6 sffeldt JE, Whittenberger JL, Mead J, Ferris BG. Pulmonary function in convalescent poliomyelitic patients. II. The pressure-volume relations of the thorax and lungs of chronic respiratory patients. $N$ Engl J Med 1952; 247:43-7.

7 Ferris BG, Whittenberger JL, Affeldt JE. Pulmonary function in convalescent poliomyelitic patients. I. Pulmonary subdivisions and maximum breathing capacity. $N$ Engl J Med 1952; 246: 919-23.

8 Kreitzer SM, Saunders NA, Tyler HR, Ingram RH Jr. Respiratory muscle function in amyotrophic lateral sclerosis. Am Rev Respir Dis 1978; 117:437-47.

9 Kilburn KH, Eagan JT, Sieker HO, Heyman A. Cardiopulmonary insufficiency in myotonic and progressive muscular dystrophy. $N$ Engl J Med 1959; 261:1089-96.

19 Braun NMT, Rochester DF. Muscular weakness and respiratory failure. Am Rev Respir Dis 1979; 119:123-5.

11 Saunders NA, Rigg JRA, Pengelly LD, Campbell EJM. Effect of curare on maximum static PV relationships of the respiratory system. J Appl Physiol 1978; 44:589-95.

12 De Troyer A, Bastenier-Geens J. Effects of neuromuscular blockade on respiratory mechanics in conscious man. J Appl Physiol 1979; 47:1162-8.

13 De Troyer A, Yernault JC, Englert M, Baran D, Paiva M. Evolution of intrathoracic airway mechanics during lung growth. J Appl Physiol 1978; 44:521-7.

14 Amrein R, Keller R, Joos H, Herzog H. Valeurs théoriques nouvelles de l'exploration de la fonction ventilatoire du poumon. Bull Physiopathol Respir 1970; 6:317-49.

15 De Troyer A, Yernault JC. Inspiratory muscle force in normal subjects and patients with interstitial lung disease. Thorax 1980; 35:92-100.

16 Gibson GJ, Pride NB. Lung mechanics in diaphragmatic paralysis. Am Rev Respir Dis 1979; 119:119-20.

17 Stubbs SE, Hyatt RE. Effect of increased lung recoil pressure on maximal expiratory flow in normal subiects. J Appl Physiol 1972; 32:32.5-31.

18 Sybrecht GW, Garret L, Anthonisen NR. Effect of chest strapping on regional lung function. J Appl Physiol 1975; 39:707-13.

19 Gibson GJ, Pride NB. Pulmonary mechanics in 
fibrosing alveolitis. The effects of lung shrinkage. Am Rev Respir Dis 1977; 116:637-47.

20 Lavietes MH, Min B, Hagstrom JWC, Rochester DF. Diffuse pulmonary granulomatous disease in the dog: relation between pressure-volume behaviour and morphologic features. Am Res Respir Dis 1977; 116:907-17.

21 Leith DE, Mead J. Mechanisms determining residual volume of the lungs in normal subjectsen J Appl Physiol 1967; 23:221-7. 